\title{
Clinical Utility of N-13 Ammonia Cardiac PET Perfusion Imaging in the Assessment of Epicardial Coronary Lesions of Intermediate Range
}

\author{
Maddury Jyotsna ${ }^{1}$, Ghanta Somasekhar ${ }^{1}$, Vatturi Venkata Satya Prabhaker Rao ${ }^{2}$, \\ RNV Kumar ${ }^{1}$, E. Jyotsna ${ }^{2}$ \\ ${ }^{1}$ Cardiology Department, Nizam's Institute of Medical Sciences, Hyderabad, India \\ ${ }^{2}$ Nuclear Medicine Department, Nizam's Institute of Medical Sciences, Hyderabad, India \\ Email: mail2jyotsna@rediffmail.com,madduryjyotsna@yahoo.com
}

Received December 6, 2011; revised February 21, 2012; accepted February 28, 2012

\begin{abstract}
Objective: To identify the high risk myocardial perfusion defects which prognosticate the future ischemic cardiac events, on Positron emission tomography (PET) myocardial perfusion imaging (MPI) of intermediate coronary stenosis (ICS) on coronary angiogram (CAG) with negative single photon emission computed tomography (SPECT) MPI in chronic stable angina (CSA) patients. Methods: For this study non-infarcted CSA patients of single vessel disease (SVD) on CAG with ICS and normal LV function were selected. In all patients with negative stress SPECT MPI, stress PET MPI was done. In both scans, extent score (ES), severity score (SS), total severity/extent score ratio (TS-ESR) and Left ventricular dilatation (LVD) were noted. Patients with Positive PET MPI were closely followed for $2 \mathrm{yr}$ and PCI was performed depending on symptoms. Results: Out of 47 study group patients, during 2 yr follow up period, 11 out of 15 patients with ischemia positive PET MPI underwent percutaneous coronary intervention (PCI). Indication for PCI was rest or persistence or recurrence of angina. Four of 15 patients remained asymptomatic. In medically managed vs. PCI done patients, mean of ES was $1.2 \pm 0.5$ vs. $2.3 \pm 1$, SS was $1.2 \pm 0.5$ vs. $5.3 \pm 1.9$, TS-ESR was 1 vs. $2.5 \pm 0.5$ which was statistically significant $(\mathrm{p}=0.01)$. Conclusion: It was inferred that subgroup of PET MPI positive ICS patients with basal PET MPI showing SS $>5$ and TS-ES R $>1.5$ with LVD during stress required close follow up as they are likely require PCI subsequently. Condensed Abstract: As negative for inducible ischemia by single photon emission computed tomography myocardial perfusion imaging (SPECT MPI) is associated with low cardiac event rate, even though positive imaging by Positron emission tomography myocardial perfusion imaging (PET MPI), in patients with intermediate Coronary stenosis (ICS) may not require immediate percutaneous coronary intervention (PCI). We followed these patients for two $2 \mathrm{yr}$ and identified that basal PET MPI showing total severity score $>5$ and total severity/ extent score ratio $>1.5$ with Left ventricular dilatation during stress required PCI subsequently.
\end{abstract}

Keywords: PET MPI; SPECT MPI; Intermediate Coronary Stenosis; Percutaneous Coronary Intervention

\section{Introduction}

Single photon emission computed tomography myocardial perfusion imaging (SPECT MPI) is a well validated modality of investigation for detection and evaluation of coronary ischemia in intermediate coronary stenosis (ICS) patients [1-3]. SPECT MPI can be used for prognosis, as negative scan patients have excellent prognosis at one $\mathrm{yr}$ [4]. Now, cardiac Positron emission tomography myocardial perfusion imaging (PET MPI) allows more accurate detection of myocardial ischemia as N13 Ammonia has greater first-pass myocardial extraction than Tc- $99 \mathrm{~m}$ tetrofosmin. In addition, PET has higher spatial resolution and allows attenuation correction and better quantification of various physiologic parameters [5]. Studies comparing SPECT MPI and PET MPI in detecting coronary ischemia have demonstrated the superiority of PET over SPECT [6-8], but not many studies have concentrated on negative SPECT MPI with positive PET patients who were followed up for coronary events. We selected patients with negative SPECT MPI with ICS, who can be followed on medical management without significant risk of coronary events. PET MPI was performed in those patients and observed for development of any coronary events requiring follow up.

\section{Materials and Methods}

The study included patients of non-infarcted chronic stable angina (CSA) with intermediate coronary stenosis 
(ICS) on coronary angiogram (CAG) with SPECT MPI negative for inducible ischemia.

CSA is defined as chest pain or discomfort that typically occurs with activity or stress with the duration more than 3 months. Intermediate coronary stenosis (ICS) is defined as diameter stenosis of $50 \%$ to $70 \%$ (used QCA - quantitative coronary angiography) on luminogram. Patients with multi vessel disease, acute or previous myocardial infarction, prior coronary revascularization, left ventricular dysfunction, other associated cardiac diseases like cardiomyopathy or valvular diseases and co morbid conditions like pulmonary, renal or hepatic dysfunction were excluded. In order to prevent metabolic trapping of $13 \mathrm{~N}$-ammonia at high flow rates, exclusion criteria included associated anemia, beriberi and systemic Arterio-venous fistulas or valvular regurgitations. Complete clinical evaluation including all risk factors, thorough clinical examination and investigations with EKG, 2D echocardiogram, coronary angiogram, SPECT MPI and PET MPI were done. Study protocol is mentioned in Figure 1.

After CAG, SPECT MPI was performed by single day stress rest protocol with $8 \mathrm{mCi}$ of Tc $99 \mathrm{~m}$ Tetrofosmin administered at $85 \%$ age related target heart rate achieved by Dobutamine infusion stress to detect ischemia. Gated SPECT images were acquired after 1 hour of injection of tracer using Siemens Dual head Gamma camera. Images acquired were processed and reconstructed using filtered back projection and Butter worth filter with cut off of 0.4 and order of 5 . Images viewed in short axis, vertical long axis and horizontal long axis. Rest study was performed with $22 \mathrm{mCi}$ of Tc $99 \mathrm{~m}$ Tetrofosmin 4 hours after stress study [9]. When SPECT MPI is negative for inducible ischemia then PET MPI was done with cyclotron produced $13 \mathrm{~N}$-ammonia. During PET MPI for both rest and stress same day protocol study $15 \mathrm{~m} \mathrm{Ci}$ was used because of 10 minutes short half life of $13 \mathrm{~N}$-ammonia. ECG Gated Imaging was started 3 - 5 min after $13 \mathrm{~N}$-ammonia injection and the scan dura-

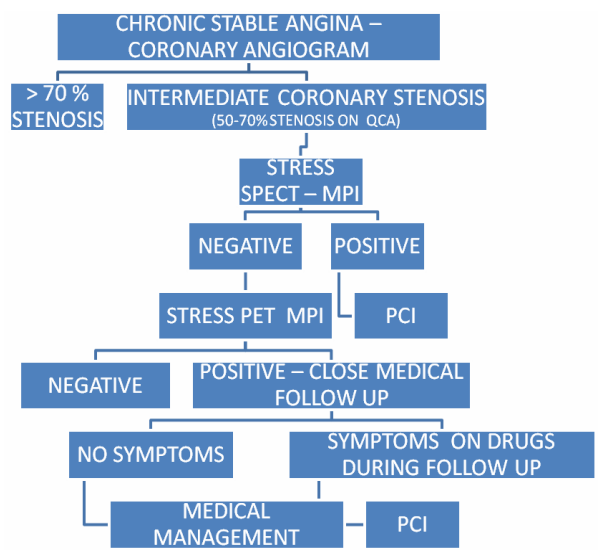

Figure 1. Study protocol. tion was approximately $20 \mathrm{~min}$. The number of gated frames were set at 8 beats with rejection of ectopic beats. Pharmacologic stress testing was performed with dobutamine infusion. The level of statistical noise in the emission and transmission images was checked to see whether enough counts have been acquired. Images viewed in short axis, vertical long axis and horizontal long axis.

Ischemic territory shown on PET MPI images were further analysed with extent score and severity apical, anterior and antero-lateral walls were classified as LAD territory segments, inferior, posterolateral, posterior, infero-lateral walls as RCA/LCX territory. Severity score was given as 0 - 3 per territory depending on degree of perfusion. $0=$ normal perfusion, $1=$ mild hypo perfusion, 2 = moderate hypo perfusion, 3 = severe to nil hypo perfusion. Total severity score and total severity/extent score ratio were also noted. The both images were assessed with a 17-segment model and a 5-point grading system by two expert observers, who were unaware of patient clinical data, angiographic findings, or other echo cardiographic results. All these patients were followed for two years. The physician making clinical decisions in these patients know the results of the PET MPI. Primary end points for need of revascularization and occurrence of coronary events were development of unstable angina or progression of symptomatic state or recurrence of symptoms following initial stabilization. Secondary end points were changes in severity of coronary stenosis on angiogram.

\section{Statistical Analysis}

Univariate analysis of categorical variables was performed with the Fisher's exact test and continuous variables were analyzed by Students' t-test. Correlations among parameters were evaluated by linear regression analysis. Differences were considered significant at $\mathrm{p}<$ 0.05. Statistical analysis was performed using SPSS software (10.0, SPSS Inc.).

\section{Results}

Eligible patients for this study were 47 . Thirteen were females. Average age was $54.4 \pm 4.5 \mathrm{yr}$.

Hypertension was present in $20(43.6 \%)$ and diabetes in $24(51.6 \%)$. Comparative demographic features of patient who had positive or negative PET scan study were mentioned in Table 1. CAG showed ICS lesion in LAD in $21(44.7 \%)$, RCA in $18(38.3 \%)$ and LCX in 8 (17\%). All LCX ICS lesion patients were negative for inducible ischemia on both PET MPI and SPECT MPI. LAD ICS in 10 patients and RCA ICS in 5 patients had positive PET MPI. The details of these patients were given in Table 2.

32 patients of ICS with PET MPI negative were fol- 
Table 1. Details of demographic features of study patients.

\begin{tabular}{cccc}
\hline Parameter & PET MPI Positive & PET MPI Negative & p value \\
\hline No of patients & 15 & 32 & - \\
Average age (yr) & $58.1 \pm 5.7$ & $52.3 \pm 3.9$ & NS \\
Hypertension (no) & 9 & 11 & NS \\
Diabetes (no) & 14 & 10 & NS \\
Dyslipedemia & 8 & 7 & NS \\
\hline
\end{tabular}

Table 2. Details of CAG and PET MPI.

\begin{tabular}{lccc}
\hline \multicolumn{1}{c}{ Parameter } & PCI group & Medical group & p value \\
\hline No of patients & 11 & 4 & 0.03 \\
Mean extent score (per patient) & $2.3 \pm 1$ & $1.2 \pm 0.5$ & 0.002 \\
Mean severity score (per territory) & $5.3 \pm 1.9$ & $1.2 \pm 0.5$ & 0.01 \\
Mean total severity/extent score (ratio) & $2.5 \pm 0.5$ & 1 & 0.00001 \\
LV dilatation during stress & 8 & 0 & \\
Culprit vessel on CAG & & 2 & \\
LAD & 8 & 2 & \\
RCA & 3 & & \\
\hline
\end{tabular}

Notes: LAD: Left anterior descending artery; RCA: Right coronary artery.

lowed up for two years with medical management (drug therapy). ICS of LAD in 11, RCA in 13 and LCX in 8 patients who had negative PET MPI. One patient developed ACS at 18 months and undergone PCI. 15 patients of ICS whose PET MPI was positive have not undergone percutaneous coronary intervention (PCI) immediately as these patients had SPECT MPI negative and were followed up closely with medical therapy. Details of these 15 patients with PET MPI positive are mentioned in Table 3. 11 out of these 15 patients had to undergo PCI subsequently. Indication for PCI was rest angina within three months in 5 patients and persistence of AP $\mathrm{Cl} 2$ symptoms on maximum dose of drugs after 3 months in 2 patients. These 7 patients showed no progression of lesion or additional thrombus at lesion site at the time of PCI. Four patients required PCI at 6, 10, 19, and 20 months. One patient presented with acute Coronary Syndrome at 6 months, one patient presented with progressive increase in severity of angina class from 1 to 3 after initial stabilization at 10 months and two patients had reappearance of AP Cl 2 symptoms on drugs at $19 \& 20$ months. All these 4 patients had progression in the severity of lesion, with or without thrombus on CAG.

Four patients with ischemia positive on PET scan remained asymptomatic at 2 yr. Figure 2 depict a case of intermediate coronary stenosis which required PCI subsequently. Mean of extent score, total severity score and total severity/extent score ratio were compared with basal
PET MPI in the 11 patients who underwent PCI with 4 patients who did not require PCI. Details of these PET scan scores were given in Table 2. This shows that the patients requiring PCI had higher extent score, severity score and severity/extent score ratio. Patients with basal PET scan showing total severity score $>5$ and total severity/extent score ratio $>1.5$ required PCI subsequently. Another important feature on basal PET scan was left ventricular dilatation, out of 11 patients who required PCI had LV dilatation during stress, but none in medical group.

\section{Discussion}

Myocardial perfusion SPECT imaging is the most commonly performed functional imaging for assessment of coronary artery disease. High diagnostic accuracy and incremental prognostic value are the major benefits while suboptimal spatial resolution and radiation exposure are the main limitations. The new SPECT imaging systems with better sensitivity, resolution, compact design and new reconstruction algorithms, the image quality has improved [10]. Additional quantification of myocardial blood flow, myocardial flow reserve noninvasively and unique ability to record changes from baseline in the left ventricular function during peak stress made cardiac PET superior to SPECT. With PET perfusion imaging, the reported average sensitivity for detecting angiographic 


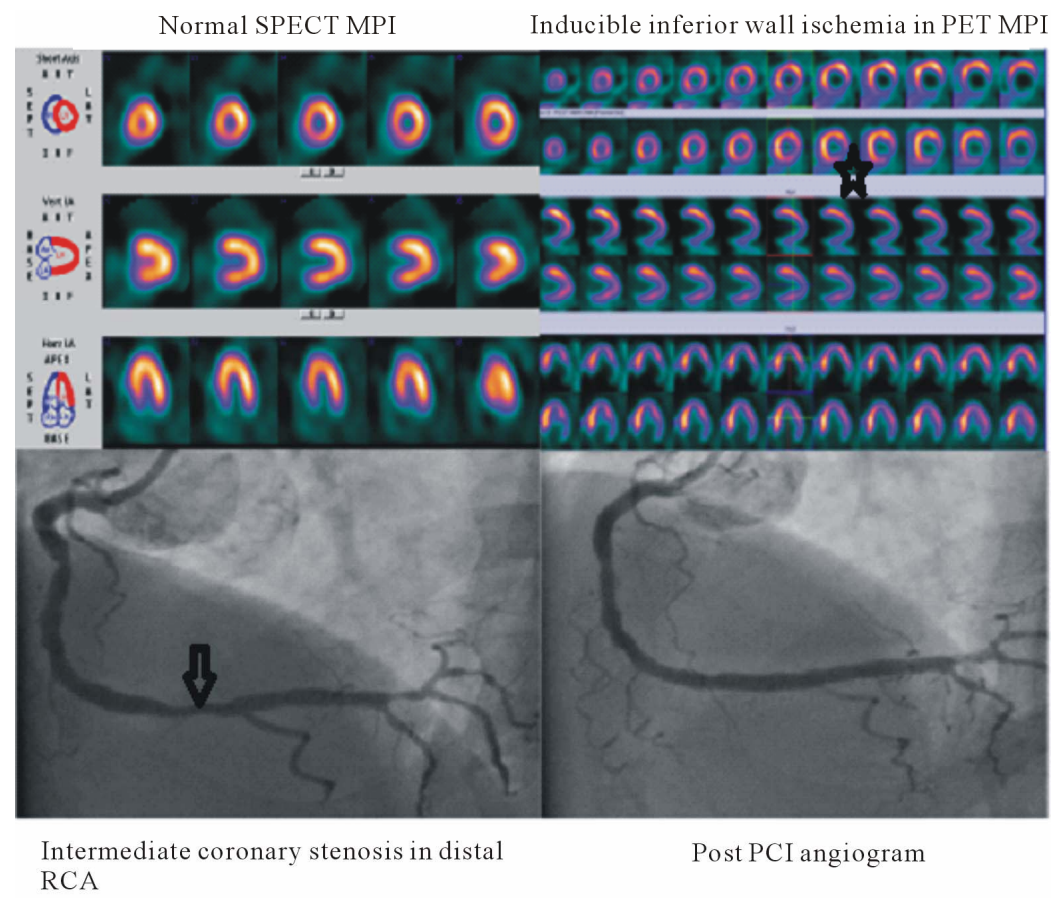

Figure 2. Coronary angiogram showing intermediate coronary stenosis in distal RCA (bottom left), post PCI angiogram (bottom right), normal SPECT MPI (top left) and inducible ischemia in PET MPI (top right) in a patient with chronic stable angina. Notes: Arrow $(\downarrow)$ is showing intermediate coronary stenosis in distal RCA, Star (*) is showing Inferior wall ischemia.

Table 3. Details of positive stress PET MPI patients.

\begin{tabular}{|c|c|c|c|c|c|}
\hline Pt. & SYM. at PRES. & Location of RI & Location of ECL & $\mathrm{M}$ of $\mathrm{FU}$ & Events During FU \\
\hline 1 & $\mathrm{AP} \mathrm{Cl} 2-3$ & $\mathrm{~A}, \mathrm{AL}, \mathrm{S}$ & Prox. LAD & $<3 \mathrm{M}$ & RA-PCI \\
\hline 2 & $\mathrm{AP} \mathrm{Cl} 2, \mathrm{Pal}$ & $\mathrm{I}, \mathrm{PL}$ & Dis. RCA & $<3 \mathrm{M}$ & RA-PCI \\
\hline 3 & $\mathrm{AP} \mathrm{Cl} 2$ & $\mathrm{AL}, \mathrm{S}$ & Mid LAD & $<3 \mathrm{M}$ & RA-PCI \\
\hline 4 & $\mathrm{AP} \mathrm{Cl} 2-3$ & $\mathrm{~A}, \mathrm{AL}, \mathrm{S}$ & LAD-D1 bif. & $<3 \mathrm{M}$ & RA-PCI \\
\hline 5 & AP Cl 2, Dys. $\mathrm{Cl} 2$ & $\mathrm{I}, \mathrm{P}$ & Mid RCA & $<3 \mathrm{M}$ & RA-PCI \\
\hline 6 & $\mathrm{AP} \mathrm{Cl} 2$ & $\mathrm{~A}, \mathrm{~S}$ & Mid LAD & $4 \mathrm{M}$ & $\mathrm{AP} \mathrm{Cl} 2-\mathrm{PCI}$ \\
\hline 7 & AP Cl 2, dys. Cl 2 & $\mathrm{AL}$ & Mid LAD & $4 \mathrm{M}$ & AP CL 2-PCI \\
\hline 8 & $\mathrm{AP} \mathrm{Cl} 2$ & I, PL & Mid RCA & $6 \mathrm{M}$ & ACS at $6 \mathrm{M}-\mathrm{PCI}$ \\
\hline 9 & $\mathrm{AP} \mathrm{Cl} \mathrm{1-2}$ & AL, Apical & Mid LAD & $10 \mathrm{M}$ & $\uparrow \mathrm{AP}$ from 1 to 3 -PCI \\
\hline 10 & $\mathrm{AP} \mathrm{Cl} 2$ & I, IL & Prox. RCA & $19 M$ & Recurrence of $\mathrm{AP} \mathrm{Cl} 2-\mathrm{PCI}$ \\
\hline 11 & $\mathrm{AP} \mathrm{Cl} 2$ & AL, Apical & Mid LAD & $20 \mathrm{M}$ & Recurrence of $\mathrm{AP} \mathrm{Cl} 2-\mathrm{PCI}$ \\
\hline 12 & $\mathrm{AP} \mathrm{Cl} 2$ & $\mathrm{AL}$ & Prox. LAD & Asym & Medical FU \\
\hline 13 & AP Cl1-2 & Apical, A & Mid LAD & Asym & Medical FU \\
\hline 14 & $\mathrm{AP} \mathrm{Cl} 2$ & IL & Mid RCA & Asym & Medical FU \\
\hline 15 & $\mathrm{AP} \mathrm{Cl} 2$ & A & Mid LAD & Asym & Medical FU \\
\hline
\end{tabular}

Notes : AP Cl: Angina pectoris class; RA: Rest angina; Pal: Palpitation; Dys: Dysponea; A: Anterior; AL: Anterio-lateral; S: Septal; I: Inferior; P: Posterior; PL: Postero-lateral; IL: Infero-lateral; Prox: Proximal; Dis: Distal; Bif.: Bifurcation; SYM: Symptoms; ECL: Epicardial Coronary lesion; RI: Regional Ischeamia; Asym: Asymptomatic; FU: Follow Up. 
stenosis of $>50 \%$ is $91 \%$ (range $83 \%-100 \%$ ) and the specificity is $89 \%$ (range $73 \%-100 \%$ ) [5,11]. Existing data supports some increased accuracy of PET over SPECT, particularly for heavier patients, where breast, chest wall, and diaphragmatic attenuation interfere with conventional SPECT. The main advantage of PET in this scenario is its ability to correct for attenuation artifacts.

Previous studies had revealed the additional ischemia detection by PET MPI which was not detectable on SPECT MPI. Our study has been designed to unearth the clinical relevance of this additional information in guiding the clinician for PCI. For this study inclusion criteria for patient selection was done keeping in mind factors which interfere with PET MPI processing and analysis. Although the sequestration of $13 \mathrm{~N}$-ammonia in the lungs is usually minimal, it may be increased in patients with depressed left ventricular (LV) systolic function or chronic pulmonary disease, thus LV dysfunction and COPD patients were excluded. To decrease the underestimation of blood flow at high flow rates, high output states were excluded [12]. Myocardial retention of $13 \mathrm{~N}$-ammonia is heterogeneous, with retention in the lateral left ventricular (LV) wall being about $10 \%$ lower than that of other segments, even in healthy subjects [13]. We have not analyzed lateral wall defect as an isolated defect in assessing the score of extent disease, unless there was involvement of inferior or posterior wall.

15 patients of stress PET MPI positive for inducible ischemia were kept on medical therapy with close follow up considering the proven fact of negative SPECT MPI having excellent prognosis. PCI was performed when patient symptomatic status progressed. Increasing extent and severity of perfusion defects on both stress SPECT MPI and PET MPI were associated with increasing frequency of adverse events $[14,15]$. Importantly, the hard event rate (i.e., myocardial infarction or cardiac death) in patients with normal stress PET was $0.4 \% / y r$. Dorbala S et al. showed that $82 \mathrm{Rb}$ myocardial perfusion PET/CT provides incremental value to clinical variables in predicting overall survival. In addition incremental prognostic value of LVEF over stress perfusion imaging was stressed [15]. So, we want to quantify the severity and extent of perfusion defect by a scoring system. This scoring system is validated as higher score were associated with need PCI subsequently. Another factor associated with need of PCI in our study was occurrence of left ventricular dilatation during stress study protocol of PET MPI. LV dilatation during stress is due to significant myocardial transient ischemia, which has an additional value over the extent score. Whether improvement or normalization of ischemic segments will translate to clinical improvement in long-term cardiovascular outcome remains clinically untested. Therefore, PET MPI was not performed in study population.

\section{Limitation of the Study}

The higher pick up rate of ischemia on PET may be a reflection of the differences in the kinetics of the flow tracers used rather than the modality (PET vs. SPECT). N13 Ammonia has one of the highest ( $>90 \%)$ first-pass myocardial extraction fractions among currently available flow tracers, and Tc-99 $\mathrm{m}$.

Tetrofosmin, the lowest ( 54\%), which became more evident by the selection criteria (that is patients with moderate, single-vessel disease and no prior infarction) to manifest differences, based on tracer extraction. But in clinical practice, this type of patients of ICS with positive PET MPI but negative SPECT MPI for inducible ischemia, requires evidence whether to intervene or not. In other subgroup of CAD patients evidence already exists for the treatment modality.

Another limitation of this study is small sample size and no blinding of the PET MPI results to the operator who takes clinical decision of patient, which may alter an acceptable outcome measures for study.

\section{Conclusion}

Patients with intermediate coronary artery stenosis on coronary angiogram without acute coronary syndrome, PET MPI findings can be used for plan of therapy. All patients positive for inducible ischemia on PET scan need not undergo PCI. Patients with PET MPI showing total severity score $<5$ and total severity/extent score ratio $<1.5$ without $\mathrm{LV}$ dilatation during stress can be followed up with drugs with minimal risk of coronary events in 2 yr. If PET MPI has total severity score $>5$ and total severity/extent score ratio $>1.5$ with LV dilatation such patients required maximum attention as they are likely to have coronary events in the near future and need revascularization.

\section{REFERENCES}

[1] F. Zijlstra, P. Fioretti, J. H. C. Reiber, et al., "Which Cineangiographically Assessed Anatomic Variable Correlates Best with Functional Measurements of Stenosis Severity? A Comparison of Quantitative Analysis of the Coronary Cineangiogram with Measured Coronary Flow Reserve and Exercise/Redistribution Thallium-201 Scintigraphy," Journal of the American College of Cardiology, Vol. 12, No. 3, 1988, pp. 686-691.

[2] J. H. O'Keefe Jr., C. S. Barnhart and T. M. Bateman, "Comparison of Stress Echocardiography and Stress Myocardial Perfusion Scintigraphy for Diagnosing Coronary Artery Disease and Assessing Its Severity," The American Journal of Cardiology, Vol. 75, No. 11, 1995, pp. 25D-34D.

[3] D. S. Berman, G. Germano and L. J. Shaw. "The Role of Nuclear Cardiology in Clinical Decision Making," Semi- 
nars in Nuclear Medicine, Vol. 29, No. 4, 1999, pp. 280297. doi:10.1016/S0001-2998(99)80017-8

[4] L. D. Metz, M. Beattie, R. Hom, R. F. Redberg, D. Grady and K. E. Fleischmann, "The Prognostic Value of Normal Exercise Myocardial Perfusion Imaging and Exercise Echocardiography: A Meta-Analysis," Journal of the American College of Cardiology, Vol. 49, No. 2, 2007, pp. 227-237. doi:10.1016/j.jacc.2006.08.048

[5] T. H. Schindler, H. R. Schelbert, A. Quercioli and V. Dilsizian, "Cardiac PET Imaging for the Detection and Monitoring of Coronary Artery Disease and Microvascular Health," JACC: Cardiovascular Imaging, Vol. 3, No. 6, 2010, pp. 623-640. doi:10.1016/j.jcmg.2010.04.007

[6] R. T. Go, T. H. Marwick, W. J. MacIntyre, et al., "A Prospective Comparison of Rubidium-82 PET and Thallium-201 SPECT Myocardial Perfusion Imaging Utilizing a Single Dipyridamole Stress in the Diagnosis of Coronary Artery Disease," Journal of Nuclear Medicine, Vol. 31, No. 12, 1990, pp. 1899-1905.

[7] R. E. Stewart, M. Schwaiger, E. Molina, et al., "Comparison of Rubidium-82 Positron Emission Tomography and Thallium-201 SPECT Imaging for Detection of Coronary Artery Disease," The American Journal of Cardiology, Vol. 67, No. 16, 1991, pp. 1303-1310. doi:10.1016/0002-9149(91)90456-U

[8] T. M. Bateman, G. V. Heller, A. I. McGhie, et al., "Diagnostic Accuracy of Rest/Stress ECG-Gated Rb-82 Myocardial Perfusion PET: Comparison with ECG-Gated Tc-99m Sestamibi SPECT," Journal of Nuclear Cardiology, Vol. 13, No. 1, 2006, pp. 24-33. doi:10.1016/j.nuclcard.2005.12.004

[9] J. Heo, J. Kegel, A. S. Iskandrrian, et al., "Comparision of Same Day Protocols Using Tc 99m Sestamibi Myocar- dial Imaging," Journal of Nuclear Medicine, Vol. 33, No. 2, 1992, pp. 186-191.

[10] M. U. Zaman, I. Hashmi and N. Fatima, "Recent Developments and Future Prospects of SPECT Myocardial Perfusion Imaging," Annals of Nuclear Medicine, Vol. 24, No. 8, 2010, pp. 565-569.

[11] M. Naya and M. F. Di Carli, "Myocardial Perfusion PET/CT to Evaluate Known and Suspected Coronary Artery Disease," The Quarterly Journal of Nuclear Medicine and Molecular Imaging, Vol. 54, No. 2, 2010, pp. 145-156.

[12] G. D. Hutchins, M. Schwaiger, K. C. Rosenspire, J. Krivokapich, H. Schelbert and D. E. Kuhl, "Noninvasive Quantification of Regional Blood Flow in the Human Heart Using N-13 Ammonia and Dynamic Positron Emission Tomographic Imaging," Journal of the American College of Cardiology, Vol. 15, No. 5, 1990, pp. 10321042. doi:10.1016/0735-1097(90)90237-J

[13] N. Tamaki, T. D. Ruddy and R. Dekamp, "Myocardial Perfusion," In: R. L. Wahl and J. W. Buchanan, Eds., Principles and Practice of Positron Emission Tomography, Lippincott Williams \& Wilkins, Philadelphia, 2002, pp. 320-333.

[14] K. Yoshinaga, B. J. Chow, K. Williams, et al., "What is the Prognostic Value of Myocardial Perfusion Imaging Using Rubidium-82 Positron Emission Tomography?" Journal of the American College of Cardiology, Vol. 48, No. 5, 2006, pp. 1029-1039.

[15] S. Dorbala, R. Hachamovitch, R. Kwong, Z. Curillova and M. F. Di Carli, "Incremental Prognostic Value of Rubidium-82 Myocardial Perfusion PET-CT Imaging in Patients with Known or Suspected CAD," Journal of the American College of Cardiology, Vol. 49, 2007, p. 109. 